\title{
Preparation of Ionic Liquid Functionalized Silica Nanoparticles for Oral Drug Delivery
}

\author{
Mehrdad Mahkam, Fatemeh Hosseinzadeh, Mohammad Galehassadi
}

Department of Chemistry, Azarbaijan Shahid Madani University, Tabriz, Iran.

Email:mmahkam@yahoo.com,mmahkam@gmail.com

Received December $24^{\text {th }}, 2011$; revised April 20 $0^{\text {th }}, 2012$; accepted June $4^{\text {th }}, 2012$

\begin{abstract}
The objective of this study is to utilize the $\mathrm{pH}$ sensitivity of modified silica nanoparticles (SNIL) by imidazole-based ionic liquid for oral delivery of insulin. In the first time, the imidazole was covalently attached to the 3-trimethoxysilylpropyl chloride with replacement of all the chlorine atoms. Then, a silica nanoparticle was modified by N-(3-trimethoxysilylpropyl) imidazole. The nanocapsule (NCIL) was achieved after the etching of the modified silica nanoparticle template with hydrofluoric acid. The nanoparticles connected through an ionic liquid-like network were characterized by FTIR and SEM. Insulin was entrapped in these carriers and the in vitro release profiles were established separately in both enzyme-free simulated gastric and intestinal fluids (SGF, pH 1) and (SIF, pH 7.4), respectively. When these drugloaded nanoparticles was placed in physiological buffer solution $(\mathrm{pH} 7.4)$, a partial negative surface charge on the modified silica nanoparticle was generated due to the deprotonation of silanol groups, and the strong electrostatic repulsion triggered a sustained release of the loaded molecules.
\end{abstract}

Keywords: Silica Nanoparticles; Nanocapsule; pH-Sensitive; Insulin; Oral Drug Delivery

\section{Introduction}

Oral delivery of drugs, especially therapeutic proteins, is the preferred route of administration because it offers advantages over injection, which is the presently accepted route of therapeutic protein administration. The oral delivery route is more natural and less invasive. The protein drug can be self-administered and the method is less expensive. However, there exist several problems for the development of oral protein delivery systems. One major problem is the degradation of proteins by proteolytic enzymes and the acidic environment of the stomach [1,2]. To reduce the non-specific absorption along the delivery path and to deliver the active form of therapeutic drug to the lower section of the gastrointestinal tract (GI tract), oral drugs should be formulated to be acid resistant to first pass through the stomach and to reduce non-specific adsorption in upper section of the intestine before the drugs can effectively reach colon tissue. Although several approaches have been studied to deliver drugs to colon tissue, their efficiency in the delivery still encounters a lot of challenges. Our response to this challenge was to design a porous nano carrier with a controlled-release function that decreased not only the degradation but also the non-specific release of drug molecules in the GI tract. Mesoporous silica materials have generated vast interest ever since they were synthe- sized by Beck and co-workers in 1992 [3]. Because of the large surface area, large pore volume, highly ordered pore structure, and adjustable pore size, mesoporous silica material have wide and interesting applications in the fields of chemical catalysts [4] and biotechnology [5]. For example, mesoporous silica material can be used as a drug carrier for the controlled release of pre-loaded therapeutic drugs [6]. Recently, mesoporous silica nanoparticles (MSN) have been synthesized using a two steps co-condensation process with cetyltrimethylammonium bromide (CTAB) as structure directing agent. Due to its nano size, MSN has much higher cellular uptake efficiency than the micron-sized mesoporous silica particle [7], thus therapeutic drugs or tracing molecules that are not easily membrane-transportable could be incorporated into the nano channels of SN to be delivered into cells.

Another advantage of MSN is it can be suspended stably in solutions. The nanochannels of MSN have been loaded with anticancer drugs to be delivered into human cancer cells to induce cell apoptosis. The MSN can also incorporate high dosages of hydrophobic drugs inside the nano channels of MSN [8]. To increase the efficiency of SN internalization into cells, SN external surface may be modified with receptor specific ligands (folic acid and lactobionic acid). The large internal surface area of SN could carry toxic drug for the rapeutic purpose. SN can 
also incorporate contrast agent for in vivo cell-tracking purpose [9]. Silica has abundant silanol groups (Si-OH) on the pore surface, which facilitate their conjugation with different functional groups to increase the adsorption and conjugation of relevant biological molecules [10]. Several research groups have studied the sustainedrelease properties of drugs loaded in conventional mesoporous silica micro sized particles (i.e., MCM-41, MCM48, and SBA-15) [11]. Vallet-Regi and co-workers [12] used aminopropyl groups modified MCM-41 with different pore sizes to regulate the release rate of ibuprofen from the siliceous matrix. Stucky and co-workers [13] also indicated that the anionic proteins could be adsorbed in the nano channels of aminopropyl group-modified mesoporous silica, and then released by changing the environmental ionic strength. In addition to amine-modified mesoporous silica, hollow mesoporous silica (HMS) nano spheres with pore channels penetrating from the outside to the inner hollow core can store significantly more aspirin molecules than conventional mesoporous silica [14]. Besides the sustained and spontaneous release system, a stimuli-responsive release system has been designed, for example, a cap formed by disulfide bond can be opened by cleavage of the disulfide bond with reducing agent to trigger the release of the entrapped molecules $[15,16]$. Xiao et al. designed a $\mathrm{pH}$ stimuli-responsive controlled release system composed of oppositely charged ionic interaction between carboxylate modified SBA-15 and cationic polyelectrolyte. When the solution condition is of mild acidity, the caps (polyelectrolyte) on the channel opening dissolve and cause loaded vancomycin to steadily release from the pores of SBA-15 [17].

Drug loading efficiency usually relies on the affinity between the nano carrier and the drug molecules. When a drug molecule is loaded inside of the non-functionalized silica matrix through a weak attraction (i.e., hydrogen bonding), a low loading capacity and a fast releasing profile are usually observed. For charge carrying drug molecules, we propose to increase the drug-loading efficiency by strengthening the electrostatic attraction through a modification of the mesoporous silica material's surface to bear more opposite charges.

In this paper, we report a synthesis of positively charged SN by attaching imidazole ionic liquid to silica nanoparticles. The nanocapsule was achieved after the etching of the modified silica nanoparticle template with hydrofluoric acid. The positive charge of ionic liquid groups generated a strong electrostatic attraction between the surface of SN and the polar groups of the drug molecule.

\section{Experimental}

The insulin used was recombinant human insulin (AK2U Nobel France; lot \# 821156, Batch L-00023822). 3- trimethoxysilylpropyl chloride and imidazole were purchased from Fluka Co. All the other chemicals used were of analytical reagent grade.

The IR spectra were recorded using a Perkin Elmer 1710 spectrophotometer. The amount of released drug was measured by a Shimadzu UV-265 FW, UV spectrophotometer at the absorption maximum of the free drug in $\mathrm{pH} 7\left(\lambda_{\max }=272 \mathrm{~nm}\right)$ using a $1 \mathrm{~cm}$ quartz cell.

\subsection{Synthesis of Silica Nanoparticles}

In a $250 \mathrm{~mL}$ round bottom flask, $60 \mathrm{~mL}(10 \mathrm{mmol}) \mathrm{am}-$ monia solution $(32 \%)$ and $1.98 \mathrm{~g}(110 \mathrm{mmol})$ water are added to $100 \mathrm{~mL}$ absolute methanol. The solution is stirred for $5 \mathrm{~min}$ before adding dropwise $10.41 \mathrm{~g}$ (500 mmol) TEOS. The final solution is stirred for three days at ambient temperature.

\subsection{Synthesize of $\mathbf{N}$-(3-Propyltrimethoxysilane) Imidazole: (NPSiI)}

The synthesis is carried out under argon atmosphere. 1Methylimidazole $(2.060 \mathrm{~g}, 25.085 \mathrm{mmol})$ and (3-chloropropyl) trimethoxysilane $(6.042 \mathrm{~g}, 25.091 \mathrm{mmol})$ were refluxed for three days at $80^{\circ} \mathrm{C}$. The orange suspension is filtered off and the solvent evacuated. By addition of 150 $\mathrm{mL}$ dry dichloromethane a precipitate appears and is filtered off under argon atmosphere. The product is then separated by distillation at $150^{\circ} \mathrm{C}$ under vacuum $(1 \mathrm{mbar})$ and $\mathrm{N}$-(3-propyltrimethoxysilane) imidazole was obtained with a honey-like consistency at room temperature (in 98\% yield) (Figure 1). ${ }^{1} \mathrm{H}$ NMR $\left(300 \mathrm{MHz}, \mathrm{CDCl}_{3}\right): \delta$ (ppm) $10.22(1 \mathrm{H}, \mathrm{NCHN}), 7.59(1 \mathrm{H}, \mathrm{NCHCH}), 7.26(1 \mathrm{H}$, $\mathrm{NCHCH}), 4.06\left(2 \mathrm{H}, \mathrm{CH}_{2} \mathrm{~N}\right), 3.86\left(3 \mathrm{H}, \mathrm{NCH}_{3}\right), 3.30(9 \mathrm{H}$, $\left.\mathrm{OCH}_{3}\right), 1.74\left(2 \mathrm{H}, \mathrm{CH}_{2} \mathrm{CH}_{2}\right), 0.37\left(2 \mathrm{H}, \mathrm{SiCH}_{2}\right)$.

\subsection{Immobilized Ionic Liquid: (SNIL)}

Then silica nanoparticles suspension are precipitated with n-hexane and extracted through centrifugation (twice at $6000 \mathrm{rpm}$ ) before being re-dissolved in dichloromethane. Silica $(1.016 \mathrm{~g})$ was suspended in $\mathrm{CH}_{2} \mathrm{Cl}_{2}(5 \mathrm{~mL})$ and NPSiI (300 mg, $0.929 \mathrm{mmol}$ ) dissolved in $\mathrm{CH}_{2} \mathrm{Cl}_{2}$ was then added. After stirring the mixture for 3 days at ambient temperature, the silica was allowed to settle. The supernatant solution was decanted and the modified silica was extracted with $\mathrm{CH}_{2} \mathrm{Cl}_{2}$ prior to being dried for several hours in vacuo (Figure 2).

\subsection{Synthesis of Nanocapsule: (NCIL)}

Immobilized ionic liquid were converted to hollow capsules by soaking composite in an aqueous solution of 12 $\mathrm{wt} \% \mathrm{HF}$ for $24 \mathrm{~h}$. The resulting products, nanocapsule, was collected by centrifugation, washed thoroughly with ethanol, and dried under vacuum. 


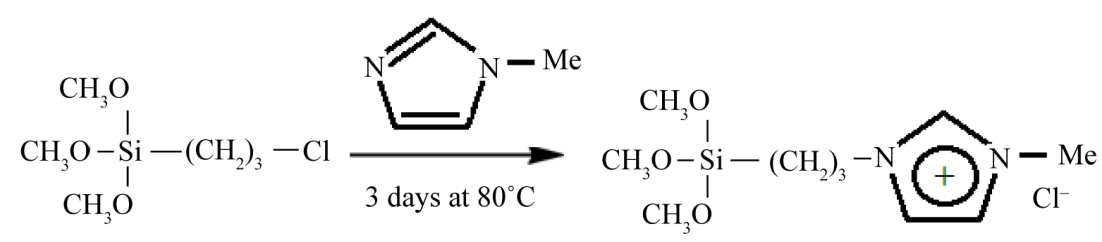

Figure 1. Synthesis of NPSiI.

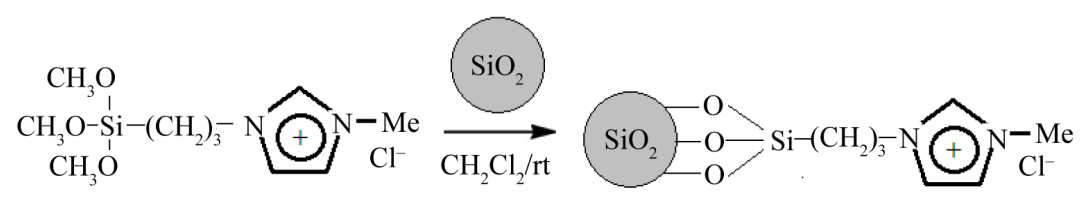

Figure 2. Synthesis of functionalized nanoparticles.

\subsection{Insulin Loading in NCIL}

One gr of each functionalized nanoparticle or nanocapsule was placed in $5 \mathrm{ml}$ of solution containing $70 \mathrm{IU}$ of insulin in $4^{\circ} \mathrm{C}$ to suck up the total amount of the drug solution. After $24 \mathrm{~h}$, the product was filtered off, washed thoroughly with distilled water and dried under vacuum at room temperature.

\subsection{Amount of Insulin Entrapped}

The amount of insulin entrapped in the nanoparticle was determined by an indirect method. After the loading, the washings with distilled water were collected and tested using UV-Vis spectroscopy. The difference between the amount of insulin initially employed and the drug content in the washings is taken as an indication of the amount of drug entrapped. The loading numbers shows in Table 1.

\subsection{Insulin Stability during Release Studies from NCIL}

In order to study the stability of insulin in contact with NCIL, two different conditions were chosen: $37^{\circ} \mathrm{C}$ and darkness, $37^{\circ} \mathrm{C}$ and light. Insulin was loaded in NCIL as described and then the peptide stability was investigated during release under the above mentioned conditions at two different $\mathrm{pH}$ values of 1 and 7.4. Samples were analyzed under each condition after 24 and $48 \mathrm{~h}$. In this condition insulin remained fairly stable at both $\mathrm{pH}$ values during the course of experiments, indicating that adsorption of the peptide to the NCIL and their release afterwards did not substantially influence the stability of this peptide drug. To investigate the protective ability of the NCIL for insulin in the harsh environment of the stomach, insulin and insulin-incorporated were treated with a simulated gastric solution that contained endopeptidase pepsin. After the treatment in gastric solution, the biological activity of insulin was determined with HPLC$\mathrm{UV}$ at $210 \mathrm{~nm}$. The flow-rate and injection volume were $1 \mathrm{~mL} / \mathrm{min}$ and $60 \mu \mathrm{L}$, respectively. Insulin was detected
Table 1. Drug loading numbers.

\begin{tabular}{cc}
\hline Nano carriers & Percent of insulin loading (\%) \\
\hline SNIL & 35 \\
NCIL & 43 \\
\hline
\end{tabular}

at a retention time of $5.5 \mathrm{~min}$ and the detection limit was $0.3 \mu \mathrm{g} / \mathrm{mL}$. These results indicated that all insulin was degraded immediately after insulin was in contact with gastric fluid and the main cause of degradation was the proteolytic enzyme, pepsin. After being treated with gastric fluid, each of NCIL demonstrated a protective effect on insulin and the biological activity remained after the treatment with gastric fluid of NCIL. Studies of NCIL showed that when the NCIL content increased, degradation of insulin decreased.

\subsection{Insulin Release from Nano Carriers}

Insulin release from the delivery systems was tested in the pyrex glasses. The powdered nanocomposite $(10 \mathrm{mg})$ was poured in $5 \mathrm{ml}$ of aqueous buffer solution $(\mathrm{pH}=1$ and 7.4) at $37^{\circ} \mathrm{C}$. The rotation speed was adjusted with stirrer. The amount of released drug was determined on UV spectrophotometer by using a standard calibration curve obtained under the same conditions.

\section{Results and Discussion}

The silica nanoparticle was stirred for $24 \mathrm{~h}$ at room temperature after HF was added. To validate the complete etching of the silica, the FTIR technique was used. In the FTIR spectrum of the products treated with HF, the absorption bands at $1105 \mathrm{~cm}^{-1}$ of the $\mathrm{Si}-\mathrm{O}-\mathrm{Si}$ symmetric stretching mode and $\mathrm{Si}-\mathrm{O}$ at $464 \mathrm{~cm}^{-1}$ disappeared. It indicated that the silica nanoparticle had been etched completely.

The hollow structure of the NCIL obtained could be observed in the SEM analysis. Figure 3 shows the SEM micrograph of the nanoparticle and nanocapsule. The 


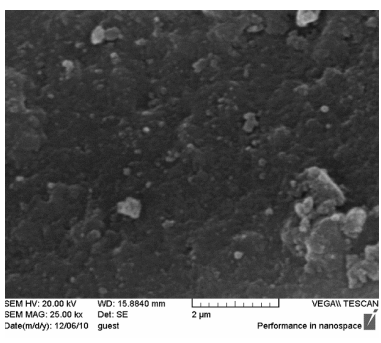

(a)

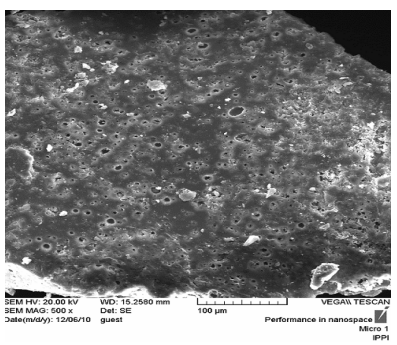

(b)
Figure 3. Scanning electron microscopic (SEM) images of SNIL (a) and NCIL (b).

average diameter of the composite particle is about 100 $\mathrm{nm}$; the particle size is also uniform.

The loading numbers in Table 1 shows which that the hollow NCIL with pore channels penetrating from the outside to the inner hollow core can store significantly more insulin molecules than conventional silica.

\section{Insulin Release}

The drug release behavior of the as prepared positive charged porous silica particles is studied to reveal their potential use in drug delivery system. In order to better mimic the vitro release of insulin from the as prepared porous silica, simulated intestinal fluid (SIF, phosphate buffersolution, $\mathrm{pH}=7.4$ ), and simulated gastric fluid ( $\mathrm{SGF}, \mathrm{HCl}$ aqueous solution, $\mathrm{pH}=1$ ) are chosen as the release fluids. A large amount of insulin could be adsorbed in a natural $\mathrm{pH}$ because the insulin, which has $\mathrm{pI}$ of 5 was negatively charge and consequently, the electrostatic attraction between the positive charge of porous silica and negative insulin molecule. The degree of hydrolysis of insulin as a function of time is shown in Figure 4. In $\mathrm{pH} 1$ and 7.4, outcome both repulsion and attraction effects in drug delivery is effective. At physiological buffer ( $\mathrm{pH}$ around 7.4), the silanol groups ( $\mathrm{Si}-\mathrm{OH})$ in the positive charge nano carrier would become deprotonated, and a strong electrostatic repulsion between the negative charges of ( $\mathrm{SiO}^{-}$groups) and the negative charge of insulin molecule would be generated. The electrostatic attraction between the drug molecules and the positive charge surface was stronger than the hydrogen bonding $\left(-\mathrm{NH}_{2} \delta-\ldots \delta+\mathrm{HOOC}-\right)$ but the weaker ionic attraction $\left(-\mathrm{NH}_{3}{ }^{+}\right.$..OOC - ). Because of the large number of deprotonated $\mathrm{SiO}^{-}$groups the amount of repulsion is a far more attractions. Consequently, the $\mathrm{pH}$ value of 7.4 in the physiological buffer promoted the releasing rate of the anionic molecules. The residual drug molecules may be occluded in the channels and therefore could not achieve the overall release.

In the simulated gastric fluid $(\mathrm{pH} 1)$, the $\mathrm{Si}-\mathrm{OH}$ groups in the positive charged nano carrier were fully protonated, so the surface predominantly carried positive charges. In

this $\mathrm{pH}$, insulin molecules have a tendency to attach to polar silanol groups due to hydrogen-bonding caused a decrease in the release rate. But the electrostatic repulsion between the positive charge of porous silica and positive insulin molecule in acidic condition caused an increase in the release rate. This time due to high volume of attraction interactions, drug release reduced. The mechanism insulin release from nano carriers is shows in (Scheme 1).

\section{Conclusion}

A pH-responsive controllable drug release system has been designed by incorporating positive charges in the framework of silica nanoparticles so that anionic molecules can be efficiently adsorbed inside of the nanochannels with minimal release under acidic $\mathrm{pH}$ value. At neutral $\mathrm{pH}$ because of the deprotonation of surface silanol groups, while giving strong electrostatic repulsion, the release rate of the adsorbed drug molecules becomes much increased. This controlled-release mechanism takes advantage of the changing $\mathrm{pH}$ value and ionic strength in our physiological buffer. Hence, this kind of the carrier could be designed as oral drug delivery system that improves site specificity and release kinetics to accommodate different therapeutic purposes.
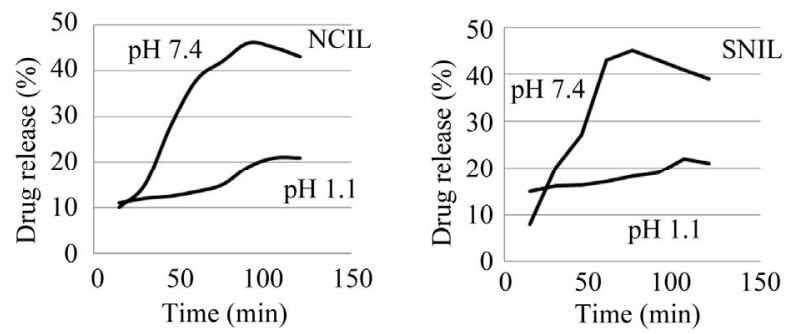

Figure 4. Release of insulin from nano carriers as a function of time at $37^{\circ} \mathrm{C}$.

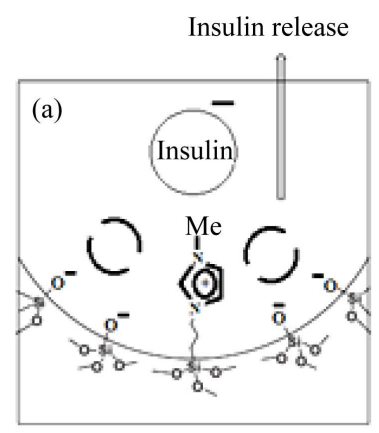

In $\mathrm{pH} 7.4$, electrostatic repulsion is more effective

Scheme 1. The representation of the release mechanism of insulin adsorbed in pH-sensitive nanocapsule. (a) In pH 7.4, electrostatic repulsion is more effective; (b) In pH 1, attraction is more effective. 


\section{Acknowledgements}

The office of research vice chancellor Azarbaijan University of Tarbiat Moallem has supported this work.

\section{REFERENCES}

[1] V. H. L. Lee and A. Yamamoto, "Penetration and Enzymatic Barriers to Peptide and Protein Absorption," Advanced Drug Delivery Reviews, Vol. 4, No. 2, 1990, pp. 171-207. doi:10.1016/0169-409X(89)90018-5

[2] J. F. Woodley, "Enzymatic Barriers for GI Peptide and Protein Delivery," Critical Reviews in Therapeutic Drug Carrier Systems, Vol. 11, 1994, pp. 61-95.

[3] J. S. Beck, M. E. Leonowicz and W. J. Roth, "Ordered Mesoporous Molecular-Sieves Synthesized by a LiquidCrystal Template Mechanism," Nature, Vol. 359, 1992, pp. 710-712. doi:10.1038/359710a0

[4] D. E. De Vos, M. Dams and B. F. Sels, "Dielectric Properties of BNT Ferroelectrics in Paraelectric Phase," Chemical Reviews, Vol. 102, 2002, pp. 3615-3640. doi:10.1021/cr010368u

[5] A. Vinu, M. Miyahara and K. Z. Hossain, "Lysozyme Adsorption onto Mesoporous Materials: Effect of Pore Geometry and Stability of Adsorbents," Journal of $\mathrm{Na}$ noscience and Nanotechnology, Vol. 7, No. 3, 2007, pp. 828-832. doi:10.1166/jnn.2007.511

[6] J. Salonen, L. Laitinen and A. M. Kaukonen, "Mesoporous Silicon Microparticles for Oral Drug Delivery: Loading and Release of Five Model Drugs," Journal of Controlled Release, Vol. 108, No. 2-3, 2005, pp. 362-374. doi:10.1016/j.jconrel.2005.08.017

[7] I. I. Slowing, B. G. Trewyn and S. Giri, "Mesoporous Silica Nanoparticles for Drug Delivery and Biosensing Applications," Advanced Functional Materials, Vol. 17, No. 8, 2007, pp. 1225-1236. doi:10.1002/adfm.200601191

[8] J. Lu, M. Liong and J. I. Zink, "Mesoporous Silica Nanoparticles as a Delivery System for Hydrophobic Anticancer Drugs," Small, Vol. 3, No. 8, 2007, pp. 13411346. doi: $10.1002 / \mathrm{smll} .200700005$

[9] J. Gu, W. Fan and A. Shimojima, "Organic-Inorganic
Mesoporous Nanocarriers, Integrated with Biogenic Ligands," Small, Vol. 3, No. 10, 2007, pp. 1740-1744. doi:10.1002/smll.200700311

[10] A. Vinu, K. Z. Hossain and K. Ariga, "Recent Advances in Functionalization of Mesoporous Silica," Journal of Nanoscience and Nanotechnology, Vol. 5, No. 3, 2005, pp. 347-371. doi:10.1166/jnn.2005.089

[11] F. Y. Qu, G. S. Zhu and S. Y. Huang, "Effective Controlled Release of Captopril by Silylation of Mesoporous MCM-41," Physical Chemistry Chemical Physics, Vol. 7, No. 2, 2006, pp. 400-406. doi:10.1002/cphc.200500294

[12] P. Horcajada, A. Ramila and J. Perez-Pariente, "Influence of Pore Size of MCM-41 Matrices on Drug Delivery Rate," Microporous and Mesoporous Mater, Vol. 68, No. 1-3, 2004, pp. 105-109. doi:10.1016/j.micromeso.2003.12.012

[13] Y. J. Han, G. D. Stucky and A. Butler, "Mesoporous Silicate Sequestration and Release of Proteins," Journal of the American Chemical Society, Vol. 121, No. 42, 1999, pp. 9897-9898. doi:10.1021/ja992138r

[14] Y. F. Zhu, J. L. Shi and Y. S. Li, "Storage and Release of Ibuprofen Drug Molecules in Hollow Mesoporous Silica Spheres with Modified Pore Surface," Microporous and Mesoporous Mater, Vol. 85, No. 1-2, 2005, pp. 75-81. doi:10.1016/j.micromeso.2005.06.015

[15] D. R. Radu, C.Y. Lai and K. Jeftinija, "A Polyamidoamine Dendrimer-Capped Mesoporous Silica Nanosphere-Based Gene Transfection Reagent," Journal of the American Chemical Society, Vol. 126, No. 41, 2004, pp. 13216-13217. doi:10.1021/ja046275m

[16] S. Giri, B. G. Trewyn and M. P. Stellmaker, "StimuliResponsive Controlled-Release Delivery System Based on Mesoporous Silica Nanorods Capped with Magnetic Nanoparticles," Angewandte Chemie International Edition, Vol. 44, 2005, pp. 5038-5044. doi:10.1002/anie.200501819

[17] F. S. Xiao, S. H. Wang and P. W. Fan, "pH-Responsive Carrier System Based on Carboxylic Acid Modified Mesoporous Silica and Polyelectrolyte for Drug Delivery," Chemistry of Materials, Vol. 17, No. 24, 2005, pp. 5999-6003. doi:10.1021/cm051198v 